\section{Synthesis of (+)-Liphagal}

Key words

enantioselective Tsuji allylation

photocycloaddition

ring expansion

olefin reduction

$\mathrm{Cu}(\mathrm{OAc})_{2} \cdot \mathrm{H}_{2} \mathrm{O}(23 \mathrm{~mol} \%)$ $\left[\mathrm{Pd}_{2}(\mathrm{dba})_{3}\right](2.5 \mathrm{~mol} \%)$ tert-butyl methyl ether, $27{ }^{\circ} \mathrm{C}$ $87 \%$ enantioselective Tsuji allylation

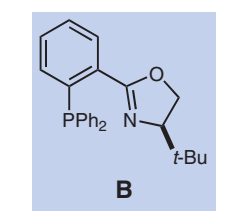<smiles>COc1cc(Br)c(C2C(=O)C3C=CC34C(C)(C)CCCC24C)cc1OC</smiles>

$\mathrm{Br}_{2}$ (1.8 equiv), $\mathrm{CHCl}_{3}$

$65 \%$

G

recryst. to er > 99.5:0.5

o-dichlorobenzene $\mathrm{MW}, 230^{\circ} \mathrm{C}, 3 \mathrm{~h}, 68 \%$<smiles>COc1cc(Br)c([C@H]2C(=O)C=CC=C3C(C)(C)CCCC32C)cc1OC</smiles>

H<smiles>C[C@H]1CC[C@H]2C(C)(C)CCC[C@]2(C)c2c1oc1c(C=O)c(O)c(O)cc21</smiles>

(+)-Liphagal
1. $\mathrm{PtO}_{2}(20 \mathrm{~mol} \%)$

$\mathrm{H}_{2}$ (1 atm), EtOAc

2. NaOMe, $\mathrm{MeOH}, 65^{\circ} \mathrm{C}, 4 \mathrm{~d}$ (3 cycles)

3. i) $\mathrm{LDA}, \mathrm{THF},-78^{\circ} \mathrm{C}$ to $0^{\circ} \mathrm{C}$ ii) $\mathrm{Mel}, \mathrm{THF},-78^{\circ} \mathrm{C}$ to $0^{\circ} \mathrm{C}$

4. DIBAL-H (4 equiv), PhMe, r.t. $33 \%$ over 4 steps

1. $\mathrm{NO}^{+} \mathrm{BF}_{4}^{-}, \mathrm{MeCN}, 0^{\circ} \mathrm{C}$

2. i) $n$-BuLi-TMEDA, THF, $0^{\circ} \mathrm{C}$ ii) $\mathrm{DMF}, 0^{\circ} \mathrm{C}$ to r.t.

3. $\mathrm{Bl}_{3}, \mathrm{CH}_{2} \mathrm{Cl}_{2}$

$22 \%$ over 3 steps

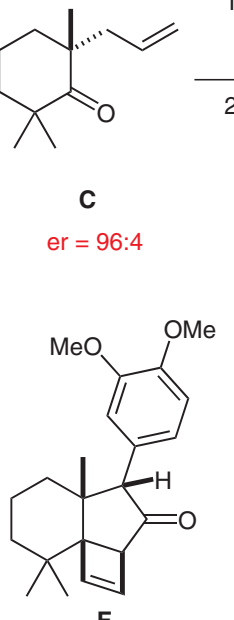

$\mathbf{F}$ $\mathrm{O}_{2}(1 \mathrm{~atm})$, DMA- $\mathrm{H}_{2} \mathrm{O}(7: 1)$

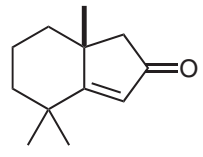

$110{ }^{\circ} \mathrm{C}$, Dean-Stark

$58 \%$ over 2 steps

D
1. $\mathrm{Me}_{3} \mathrm{SiCCH}, \mathrm{MeCN}$-acetone $(5: 1)$ UV-B lamp ( 313 nm), $22.5 \mathrm{~h}$ 2. $\mathrm{BF}_{3} \cdot \mathrm{OEt}_{2}, \mathrm{CH}_{2} \mathrm{Cl}_{2}$ 3. TBAF (2 equiv), THF, $40^{\circ} \mathrm{C}$ $69 \%$ over 3 steps

$\mathrm{Pd}\left[\mathrm{P}(t-\mathrm{Bu})_{3}\right]_{2}(5 \mathrm{~mol} \%)$ $\mathrm{NaO} t-\mathrm{Bu}$ (1.2 equiv)

4-bromoveratrole (1.1 equiv) THF, MW, $120^{\circ} \mathrm{C}, 7.5 \mathrm{~h}$ $67 \%$

single diastereomer
E
Significance: (+)-Liphagal was isolated in 2006 from the Caribbean sponge Aka coralliphaga and belongs to a family of phosphatidylinositol 3-kinase (PI3K) inhibitors. Structurally, (+)-liphagal consists of an unprecedented [6-7-5-6] tetracyclic motif. Herein, the first catalytic enantioselective approach is reported.

SYNFACTS Contributors: Steven V. Ley, Sean Newton Synfacts 2011, 10, 1039-1039 Published online: 20.09.2011 DoI: 10.1055/s-0030-1261144; Reg-No.: N05411SF
Comment: This synthesis utilizes an enantioselective Tsuji-type allylation developed within the Stoltz group and a two-step oxidation-cyclization procedure to yield previously reported enone D with good enantioselectivity. Epimerization at C10 followed by cyclization enables hydrogenation of the trisubstituted olefin, yielding the trans-fused ring as the only product. 